\title{
Purification of genuine multipartite entanglement
}

\author{
Marcus Huber ${ }^{1}$, Martin Plesch ${ }^{2,3}$ \\ ${ }^{1}$ Faculty of Physics, University of Vienna, Vienna, Austria \\ ${ }^{2}$ Faculty of Informatics, Masaryk University, Brno, Czech Republic and \\ ${ }^{3}$ Institute of Physics, Slovak Academy of Sciences, Bratislava, Slovakia
}

(Dated: 12 May 2011)

\begin{abstract}
In tasks, where multipartite entanglement plays a central role, state purification is, due to inevitable noise, a crucial part of the procedure. We consider a scenario exploiting the multipartite entanglement in a straightforward multipartite purification algorithm and compare it to bipartite purification procedures combined with state teleportation. While complete purification requires an infinite amount of input states in both cases, we show that for an imperfect output fidelity the multipartite procedure exhibits a major advantage in terms of input states used.
\end{abstract}

PACS numbers: 03.67.Ac, 42.50.Dv

\section{INTRODUCTION}

Entanglement is one of the most striking phenomena of quantum physics. While the primary interest was focused mainly on the two party case (for an overview see e.g. Ref. [1]), recent results show that with a growing system size multipartite entanglement is of great relevance in contemporary quantum theory (for an overview see e.g. Ref. [2]).

Entanglement is inherently manifested in various physical systems, reaching from condensed matter systems (see e.g. Ref. 3]), over ionization of quantum gases (see e.g. Ref. [4]) to small biological systems (see e.g. Refs. [5, 6]). Also in quantum information processing (QIP) tasks it is in many cases at the heart of their advantage over the respective classical counterparts. It is the crucial resource for measurement based quantum computing (see e.g. Ref. 7] ), is involved in most of the popular quantum algorithms (see e.g. Ref. [8]) and enables multiparty quantum communication (see e. g. Refs. 9, 10]).

Recent advances have made it possible to discriminate genuinely multipartite entangled states from partially separable ones (see e.g. Refs. [11 15]). Some of these criteria are in large systems implementable even in an experimentally feasible way and are very robust against noise (see e.g. 16 19]). This verification enables secure multipartite quantum communication protocols such as quantum secret sharing (see e.g. Refs. [9, 20]). This protocol however refers to perfectly noiseless and error free communication procedures, which even at medium distances and realistic lab conditions can never be achieved. Therefore a purification (also referred to as distillation in other contexts) procedure was developed, where several noisy copies of the shared state are employed in an iterative algorithm to reduce the noise of a subset of these. If the purification succeeds, eavesdropping and cheating can be excluded with certainty, thus providing secure quantum communication even in realistic lab conditions.

Surprisingly, the purification of genuine multipartite entanglement does not require the state of the system to exhibit any genuine multipartite entanglement at all (i.e. is possible for states which can be decomposed as a convex sum of biseparable pure states). As long as one party is able to purify bipartite entanglement with respect to all other parties, it is possible two create any kind of genuinely multipartite entangled states by means of quantum teleportation (as pointed out in Ref. [2]). Multipartite purification procedures of states affected by bipartite noisy channels have been intensively investigated in Refs. [21 27] and general distillation of multipartite entanglement has been studied in Refs. [28 30].

In this paper we consider a generalized purification algorithm for multipartite states, which is based on the procedure originally introduced in Ref. 31 and is similar to the algorithm introduced in Ref. [32]. In our setting parties share a multipartite (imperfectly) entangled state, which can be a result of a previously obtained state, which has undergone decoherence, or a state, which was transferred via a noisy multipartite channel. We compare the efficiency of this protocol with its bipartite counterpart in the means of the number of resource states used to get a single, highly entangled output state.

Let us begin by concisely defining the setup: $\mathrm{N}$ parties (among them Dora the dealer) wish to implement a QIP task employing genuine multipartite entanglement. They have prepared and shared a genuinely multipartite entangled state $|G H Z\rangle=\frac{1}{\sqrt{2}}\left(|0\rangle^{\otimes N}+|1\rangle^{\otimes N}\right)$, also known as GHZ (Greenberger-Horne-Zeilinger) state (this specific state is e.g. used in the quantum secret sharing protocols in Refs. [9, 20]). However, due to decoherence or noise in the multipartite channel used for distributing the qubits the parties end up with a noisy mixed state

$$
\rho_{i n}=q|G H Z\rangle\langle G H Z|+\frac{1-q}{2^{N}} \mathbb{1} .
$$

The fidelity of this state with a pure GHZ state is $F_{i n}=q+\frac{(1-q)}{2^{N}}=\frac{\left(2^{N}-1\right) q+1}{2^{N}}$ and in-fidelity $\delta=1-F_{i n}=$ $\frac{\left(2^{N}-1\right)}{2^{N}}(1-q)$. This fidelity might not be sufficient for performing the QIP task (e.g. secure quantum secret sharing) reliably. So the parties have to use more copies of the state (11) and perform purification on them to obtain a single, high quality, state. We will express the effectiveness of the protocol as the number of source multipartite states (1) needed to obtain a single highly entangled state with a fidelity at least $1-\varepsilon$. 


\section{BIPARTITE PROCEDURE}

We proceed as follows: Dora prepares bipartite entangled states with all other parties. Secondly, Dora performs bipartite distillation protocols with all other parties to obtain pure enough states for stage three. In this stage, Dora locally prepares a GHZ state of $N$ qubits and use the bipartite entangled states to teleport all the qubits of this state but one to all other parties.

\section{Preparation of the bipartite state}

Let us assume that Dora wants to prepare a bipartite entangled state with one of the other parties, Julia. She selects one of the source states and asks all other parties to perform a measurement in the $| \pm\rangle$ basis. Depending on the outcomes of the measurements of the other parties, the bipartite state shared between Dora and Julia will be $\rho_{D J}=q\left|\Phi^{ \pm}\right\rangle\left\langle\Phi^{ \pm}\right|+\frac{1-q}{4} \mathbb{1}$, where $\left|\Phi^{ \pm}\right\rangle=\frac{1}{\sqrt{2}}(|00\rangle \pm|11\rangle)$ are Bell states and the sign is given by the parity of outcomes of the state $|-\rangle$ of the measurement. Dora collects all the information about the measurement outcomes from all parties except Julia and in the case of an odd number of the $|-\rangle$ results she will perform a local phase operation to correct the sign. In such a way Dora prepares $M$ bipartite states with each of the $N-1$ remaining parties, requiring $M(N-1)$ source states.

\section{Bipartite distillation}

As a second step, Dora performs distillation of entanglement on all $M$ bipartite entangled pairs shared with Julia. Using the procedure described in [31], in every step they can concentrate entanglement from two pairs to one pair by performing a C-NOT operation on pairs of qubits on both sides and measuring the target qubits. After this operation, the parameter $q$ changes to

$$
q \rightarrow \frac{4 q^{2}+2 q}{3\left(q^{2}+1\right)}
$$

Dora and Julia have used two pairs of bipartite source states between them and the result 0 is obtained with probability $\frac{1}{2}$ on Doras' side. The probability of measuring 0 in Julias' side depends on the parameter $q$ and is $\frac{1+q}{2}$. Thus, for every step they need in average $\frac{8}{1+q}$ source states, which can be bounded from below by 4 . With $k$ steps for all parties, they have used more than $4^{k}(N-1)$ states to obtain $N-1$ pairs of highly entangled states with the parameter $q_{k}$ given by the recursive application of the formula (2).

\section{Reexportation}

We use the distilled states to perform reexportation 33 of individual qubits of a GHZ state locally prepared by Dora. As the shared entanglement is not perfect, neither will the reexportation be. Dora performs a Bell measurement on a pair of qubits, where one qubit will be taken from the GHZ state and the other from the bipartite state shared with Julia. She communicates the result consisting of two bits to Julia and Julia performs corrective transformation, if needed. After repeating this procedure with all other parties, the shared state will have the form

$$
\begin{gathered}
\rho_{f}=q_{k}^{N-1}|G H Z\rangle\langle G H Z|+ \\
\sum_{j=1}^{N-1}\left(1-q_{k}\right)^{j} q_{k}^{N-j-1} \sum_{i=1}^{\left(N_{j}^{N-1}\right)} 2^{-j} \mathbb{1}_{B(j, i)} \otimes \rho_{\text {rest }}^{\text {diag }} \\
\rho_{s}^{\text {diag }}=\frac{1}{2}\left(|0\rangle\left\langle\left. 0\right|^{\otimes s}+\mid 1\right\rangle\left\langle\left. 1\right|^{\otimes s}\right),\right.
\end{gathered}
$$

where $B(j, i)$ is a set of $j$ qubits, selected by the choice of $i$ and we sum over all possible sets of qubits. The fidelity of this state with the GHZ state is

$$
\begin{aligned}
F_{\text {out }} & =q_{k}^{N-1}+\sum_{j=1}^{N-1}\left(1-q_{k}\right)^{j} q_{k}^{N-j-1}\left(\begin{array}{c}
N-1 \\
j
\end{array}\right) 2^{-j-1} \\
& =\frac{1}{2}\left[q_{k}^{N-1}+\left(\frac{1+q_{k}}{2}\right)^{N-1}\right]
\end{aligned}
$$

and is expected to be at least $1-\varepsilon$.

\section{Small errors}

We will analyze the results for the case when $\delta$ is small enough (hence $q$ close to 1 ). Substituing $\Delta=1-q$, the transformation formula for distillation (2) changes to

$$
\Delta \rightarrow \frac{4 \Delta-4 \Delta^{2}}{6-6 \Delta+3 \Delta^{2}}
$$

which simplifies to $\Delta \rightarrow \frac{2}{3} \Delta$. Substituing back we get $q \rightarrow \frac{1}{3}(1+2 q)$ and we can calculate the recurrence directly obtaining (after $k$ distillation steps) $q_{k}=1-$ $\left(\frac{2}{3}\right)^{k}(1-q)$. Substituting to the approximated version of (3) we get

$$
(N-1)(1-q)\left(\frac{2}{3}\right)^{k-1} \leq \varepsilon
$$

and

$$
k \geq 1+\log \left[\frac{2^{N}}{2^{N}-1} \frac{\delta}{\varepsilon}(N-1)\right] / \log \left[\frac{3}{2}\right]
$$

with logarithm taken in basis 2 . The overall number of states needed will thus be at least

$$
4(N-1)\left[\frac{2^{N}}{2^{N}-1} \frac{\delta}{\varepsilon}(N-1)\right]^{\frac{2}{\log [3 / 2]}},
$$


which can be approximated for large $N$ as

$$
4 N^{4.42}\left(\frac{\delta}{\varepsilon}\right)^{3.42}
$$

\section{Large errors}

For larger errors the approximations do not work and it is not possible to calculate an explicit expression for the recurrence formula (2). However, numerical analysis for different values of $\delta$ and $N$ for a fixed $\varepsilon=0,01$ show a strong dependence of the number of source states needed on $N$.

\section{Smart distillation}

More advanced distillation protocols mentioned in Ref. 31] suggest that it might be possible to perform more effective protocols, if the fidelity of the input state with the expected entangled state is known. In this case, the fraction of states lost in every distillation step depends on the fidelity of the source states and might be rather low. But even if we had allowed Dora and Julia to use a virtually ideal distillation protocol, allowing them to extract all bipartite entanglement from the shared state (given by the entanglement of formation), the bipartite procedure would still have been highly inefficient due to large loss on fidelity given by (3).

\section{MULTIPARTITE PROCEDURE}

In the multipartite case, all participants employ local operations and classical communication (LOCC) to purify $\rho_{i n}$ directly. The generalization of the bipartite distillation procedure introduced in Ref. [31] works as follows:

- All parties take two copies of the input state

- They label the first qubit target and the second control and perform a CNOT-gate operation on their two qubits

- They measure their target qubit in $\sigma_{z}$ basis, keep their control qubit if the outcome is 0 , and dismiss it otherwise

- Finally they communicate their results, and only keep those states, where they all shared the outcome 0 and dismiss all others. In this case the state exhibits stronger multipartite entanglement than the source state.

Except for the fact that multipartite distillation directly uses multipartite entanglement provided in the source, without converting it to bipartite entanglement and back, it can also by performed in a much simpler way, compared to the bipartite one Ref. 31]. Randomized rotations between every iteration step are not necessary, if at least three-partite entanglement is distilled.

\section{Distillation operation}

We define the map

$$
\Lambda\left[\rho^{\otimes 2}\right]:=\operatorname{Tr}_{c_{1} c_{2} \cdots c_{n}}\left[D \rho^{\otimes 2} D^{\dagger}\right]
$$

with

$$
D:=(\mathbb{1} \otimes|0\rangle\langle 0|)^{\otimes n} C N O T^{\otimes n},
$$

where the partial trace is taken over all target qubits. As

$$
\begin{aligned}
\Lambda\left[\mathbb{1}^{\otimes 2}\right] & =\mathbb{1} \\
\Lambda\left[|G H Z\rangle\left\langle\left. G H Z\right|^{\otimes 2}\right]\right. & =\frac{1}{2}|G H Z\rangle\langle G H Z| \\
\Lambda[|G H Z\rangle\langle G H Z| \otimes \mathbb{1}] & =\Lambda[\mathbb{1} \otimes|G H Z\rangle\langle G H Z|]=\rho_{n}^{\text {diag }},
\end{aligned}
$$

the initial state

$$
\rho_{i n}=q|G H Z\rangle\langle G H Z|+\frac{1-q}{2^{N}} \mathbb{1}
$$

transforms to

$\Lambda\left[\rho^{\otimes 2}\right]=\frac{q^{2}}{2}|G H Z\rangle\langle G H Z|+\frac{(1-q)^{2}}{2^{2 N}} \mathbb{1}+\frac{2 q(1-q)}{2^{N}} \rho_{n}^{\text {diag }}$.

The success probability of this procedure (probability of obtaining the result 0 from measurements of all parties) depends on the initial state and is given by:

$$
P_{\text {succ }}=\operatorname{Tr}\left[\Lambda\left[\rho^{\otimes 2}\right]\right]=\frac{q^{2}}{2}+\frac{2 q(1-q)}{2^{N}}+\frac{(1-q)^{2}}{2^{N}}
$$

For large $N$ it is always sufficient to apply the protocol once and the result is already arbitrarily close to the GHZ state. For intermediate $N$, however, it might be necessary to repeat the procedure more times. In such a case the input state will be of a different form

$$
\rho_{i}=q|G H Z\rangle\langle G H Z|+\frac{r}{2^{N}} \mathbb{1}+s \rho_{n}^{\text {diag }} .
$$

Using the additional relations

$$
\begin{aligned}
\Lambda\left[\mathbb{1} \otimes \rho_{n}^{\text {diag }}\right] & =\Lambda\left[\rho_{n}^{\text {diag }} \otimes \mathbb{1}\right]=\rho_{n}^{\text {diag }} \\
\Lambda\left[|G H Z\rangle\langle G H Z| \otimes \rho_{n}^{\text {diag }}\right] & =\Lambda\left[\rho_{n}^{\text {diag }} \otimes|G H Z\rangle\langle G H Z|\right]=\frac{1}{2} \rho_{n}^{\text {diag }} \\
\Lambda\left[\rho_{n}^{\text {diag }} \otimes \rho_{n}^{\text {diag }}\right] & =\frac{1}{2} \rho_{n}^{\text {diag }}
\end{aligned}
$$

we can continue the iteration using recurrence relations

$$
q \rightarrow \frac{q^{2}}{2}, r \rightarrow \frac{r^{2}}{2^{N}}, s \rightarrow \frac{s^{2}+s q}{2}+\frac{s r+2 q r}{2^{N}}
$$

until we reach the desired fidelity with the $G H Z$-state. This result implies that the $G H Z$-state remains distillable up to a noise threshold of $q_{\text {noise }}=\frac{2}{2^{n}-2}$. This is remarkable as it was recently shown using semidefinite programming that e.g. the four party $G H Z$-state is biseparable up to a noise threshold of $q_{\text {noise }} \approx 0.46$ in Ref. 34. 


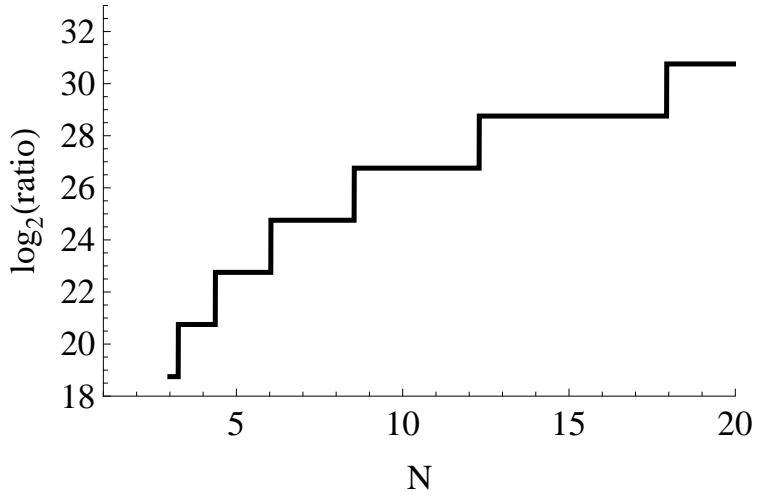

FIG. 1: Logarithm (base 2) of the ratio between the number of source states with in-fidelity $\delta=0.2$ needed to get a single GHZ state with in-fidelity $\varepsilon=0.01$ for different values of $N$, using bipartite and multipartite distillation. The ratio even for moderate values of $N$ exceeds $10^{6}$.

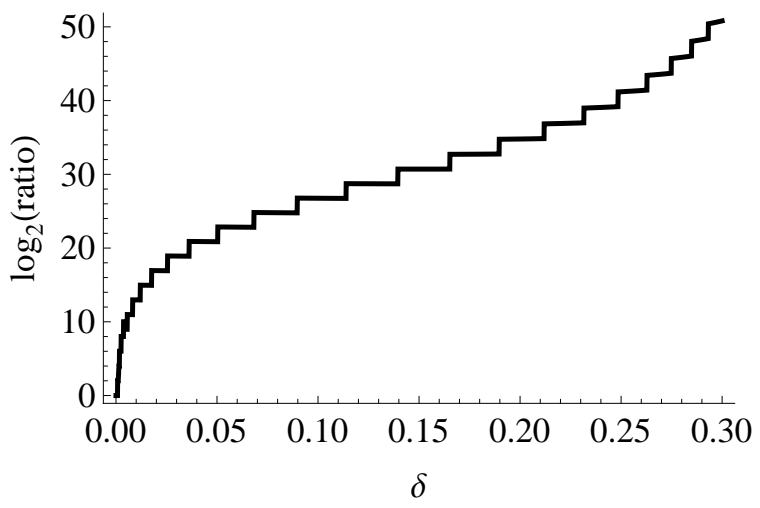

FIG. 2: Logarithm (base 2) of the ratio between the number of source states needed to get a single GHZ state with infidelity $\varepsilon=0.01$ for 10 parties and different values of $\delta$, using bipartite and multipartite distillation.

The number of resource states needed for the whole procedure is then given by the inverse of the norm of the state (6) $M=\frac{1}{(q+r+s)}$. In Figures 1 and 2 we show the ratio of the number of resource states needed to reach a given output fidelity of a single state. It can be clearly seen that the multipartite procedure is far more effective even for a moderate number of parties $N$ and for the whole scale of input fidelities. What is especially important, the distillation protocol produces high-fidelity GHZ states after a single, or a few steps even in the case of very low input fidelities (e.g. $\delta=0.5$ ), where the bipartite distillation protocol is completely useless.

\section{CONCLUSIONS}

In this paper we have shown an example of a scenario, in which direct multipartite entanglement purification is far more efficient than bipartite distillation protocols combined with state teleportation. For a moderate num- ber of parties the multipartite protocol gives reasonable results even with extremely high input noise (e.g. reaching up to $\approx 99.8 \%$ for $n=10$ ). The amount of resources needed using the bipartite protocol grows much faster with the input noise than in the multipartite case. The nature of the advantage stems mainly from the structure of the noise, which has inherently multipartite features.

Furthermore, this paper provides evidence of biseparable states being distillable to genuinely multipartite entangled ones without resorting to state teleportation.

In a different context, using bipartite noisy communication channels, there has been intensive study of a very similar scenario in Refs. 21, 24, 25, 27], also comparing the effectiveness of the two different strategies. In this case there is a noise regime where the multipartite purification approach still outperforms the bipartite one, however, the advantage never reaches the level obtained for multipartite channels. This leads to a surprising conclusion that multipartite entangled states affected by noise in multipartite channels are far easier and more effectively purifiable than the same states affected by local noise.

\section{ACKNOWLEDGMENTS}

We thank Ch. Spengler and A. Gabriel for inspiring discussions. This work was supported by the SoMoPro project SIGA 862 and by the CE SAS QUTE. The collaboration is a part of ÖAD/APVV SK-AT-0015-10 project. M. Huber gratefully acknowledges the support of the Austrian Fund project FWF-P21947N16.

[1] R. Horodecki, P. Horodecki, M. Horodecki and K. Horodecki, Rev. Mod. Phys. 81, 865-942, (2009).

[2] O. Gühne and G. Toth, Phys. Rep. 474, 1 (2009).

[3] S. Sachdev, Quantum Phase Transitions, (Cambridge University Press, Camebridge, England, 1999).

[4] D. Akoury et al., Science 9 Vol. 318. no. 5852, p. 949 952, (2007).

[5] F. Caruso, A.W. Chin, A. Datta, S.F. Huelga and M.B. Plenio, J. Chem. Phys. 131, 105106 (2009).

[6] M. Sarovar, A. Ishizaki, G. R. Fleming and K. B. Whaley, Nature Physics, 6, 462 (2010).

[7] R. Raussendorf and H.-J. Briegel, Phys. Rev. Lett. 86, 5188 (2001).

[8] D. Bruss, C. Macchiavello, arXiv/quant-ph:1007.4179.

[9] M. Hillery, V. Buzek, and A. Berthiaume, Phys. Rev. A 59, 1829 (1999).

[10] D. Markham and B. C. Sanders, Phys. Rev. A 78, 042309 (2008).

[11] M. Horodecki, P. Horodecki and R. Horodecki, Phys. Lett. A 283, 1 (2001).

[12] P. Wocjan and M. Horodecki, Open Syst. Inf. Dyn. 12, 331 (2005).

[13] C.S. Yu and H.S. Song, Phys. Rev. A 72, 022333 (2005).

[14] A. Hassan and P. Joag, Quantum Inf. Comput. 8, 8\&9, 0773-0790 (2008).

[15] B.C. Hiesmayr, M. Huber and Ph. Krammer, Phys. Rev. A. 79, 062308 (2009). 
[16] M. Seevinck and J. Uffink, Phys. Rev. A 78, 032101 (2008).

[17] O. Gühne and M. Seevinck, New J. Phys. 12, 053002 (2010).

[18] M. Huber, F. Mintert, A. Gabriel, B. C. Hiesmayr, Phys. Rev. Lett. 104, 210501 (2010).

[19] A. Gabriel, B. Hiesmayr and M. Huber, QIC 10, 08290836 (2010).

[20] S. Schauer, M. Huber, B. C. Hiesmayr, Phys. Rev. A 82, 062311 (2010)

[21] W. Dür, H. Aschauer and H.-J. Briegel, Phys. Rev. Lett. 91, 107903 (2003).

[22] H. Aschauer, W. Dür and H.-J. Briegel, Phys. Rev. A 71, 012319 (2005)

[23] A. Kay, J. Pachos, W. Dür and H.-J. Briegel, New J. Phys. 8, 147 (2006).

[24] C. Kruszynska, A. Miyake, H. J. Briegel and W. Dür, Phys. Rev. A 74, 052316 (2006).

[25] W. Dür and H.-J. Briegel, Rep. Prog. Phys. 70, 1381 (2007).
[26] W. Dür and J. I. Cirac, Phys. Rev. A 61, 042314 (2000).

[27] C. Kruszynska, S. Anders, W. Dür, and H. J. Briegel, Phys. Rev. A 73, 062328 (2006).

[28] M. Murao, M. B. Plenio,S. Popescu, V. Vedral, and P. L. Knight, Phys. Rev. A 57, R4075 (1998).

[29] K. Chen, H.-K. Lo, Quant. Inf. \& Comp. Vol. 7, No.8, 689-715 (2007).

[30] E. Maneva and J. Smolin, AMS Contemp. Math. Ser., Vol. 305, p. 203-212, (2002).

[31] Ch. H. Bennett, G. Brassard, S. Popescu, B. Schumacher, J. A Smolin, and W. K. Wootters, Phys. Rev. Lett. 76, 722 (1996).

[32] R. Augusiak and P. Horodecki, Phys. Rev. A 80, 042307 (2009).

[33] C. H. Bennett, G. Brassard, C. Crepeau, R. Jozsa, A. Peres, and W. K. Wootters, Phys. Rev. Lett 70, 1895 (1993).

[34] B. Jungnitsch, T. Moroder, O. Gühne, Phys. Rev. Lett. 106, 190502 (2011). 